(C) The Authors 2017. This is an Open Access article, distributed under the terms of the Creative Commons Attribution licence (http:// creativecommons.org/licenses/by/4.0/), which permits unrestricted re-use, distribution, and reproduction in any medium, provided the original work is properly cited.

\title{
Cultural effects on neurodevelopmental testing in children from six European countries: an analysis of NUTRIMENTHE Global Database
}

\author{
Miguel Pérez-García ${ }^{1}$, Juan de Dios Luna ${ }^{2}$, Francisco J. Torres-Espínola ${ }^{3}$, Cristina Martínez-Zaldívar ${ }^{3}$, \\ Tania Anjos ${ }^{3}$, Jolien Steenweg-de Graaff ${ }^{4}$, Martina Weber ${ }^{5}$, Veit Grote ${ }^{5}$, Dariusz Gruszfeld ${ }^{6}$, \\ Elvira Verduci ${ }^{7}$, Pascale Poncelet ${ }^{8}$, Joaquín Escribano9 ${ }^{9}$, Henning Tiemeier ${ }^{4}$, Berthold Koletzko ${ }^{5}$ and \\ Cristina Campoy ${ }^{3,10 *}$ \\ ${ }^{1}$ Mind, Brain and Behaviour Research Center (CIMCYC), University of Granada, Campus de Cartuja, 18071 Granada, Spain \\ ${ }^{2}$ Department of Biostatistics, University of Granada, Avda de la Investigación 11, 18016 Granada, Spain \\ ${ }^{3}$ Excellence Centre for Paediatrics Research (EURISTIKOS), University of Granada, Avda de la Investigación 11,18016 \\ Granada, Spain \\ ${ }^{4}$ The Generation $R$ Study Group, Erasmus University Medical Centre, PO Box 2040, 3000 CA Rotterdam, The Netherlands \\ ${ }^{5}$ Division of Metabolism and Nutritional Medicine, Dr von Hauner Children's Hospital, Ludwig-Maximilians-University of \\ Munich Medical Centre, Lindwurmstr, 4, D-80337 Munich, Germany \\ ${ }^{6}$ Neonatal Intensive Care Unit, Department of Gastroenterology, Hepatology and Immunology, Children's Memorial Health \\ Institute, Al. Dzieci Polskich 20, 04-736 Warsaw, Poland \\ ${ }^{7}$ Department of Paediatrics, San Paolo Hospital, University of Milan, 8 Via A di Rudini, 20142 Milano, Italy \\ ${ }^{8}$ Department of Paediatrics, Centre Hospitalier Chrétien (CHC) St Vincent, 4000 Liège-Rocourt, Belgium \\ ${ }^{9}$ Pediatrics Research Unit, Institut d'Investigació Sanitària Pere Virgili (IISPV), Universitat Rovira I Virgili, 43201 Reus, Spain \\ ${ }^{10}$ Department of Paediatrics, University of Granada, Avda de la Investigación 11, 18016 Granada, Spain
}

(Submitted 1 July 2015 - Final revision received 12 October 2016 - Accepted 28 October 2016 - First published online 7June 2017)

\section{Abstract}

Cultural background is an important variable influencing neuropsychological performance. Multinational projects usually involve gathering data from participants from different countries and/or different cultures. Little is known about the influence of culture on neuropsychological testing results in children and especially in European children. The objectives of this study were to compare neuropsychological performance of children from six European countries (Belgium, Germany, Italy, The Netherlands, Poland and Spain) using a comprehensive neuropsychological battery and to apply a statistical procedure to reduce the influence of country/cultural differences in neuropsychological performance. As expected, the results demonstrated differences in neuropsychological performance among children of the six countries involved. Cultural differences remained after adjusting for other confounders related to neuropsychological execution, such as sex, type of delivery, maternal age, gestational age and maternal educational level. Differences between countries disappeared and influence of culture was considerably reduced when standardised scores by country and sex were used. These results highlight the need for developing specific procedures to compare neuropsychological performance among children from different cultures to be used in multicentre studies.

Key words: Neuropsychological testing: Cultures: Children: Diets: Mental performance

Neuropsychological assessment during infancy and childhood are frequently implemented as outcomes in nutrition research because of the consistent link between some nutrients with brain development and neuropsychological functioning ${ }^{(1-4)}$. Investigating this relationship in an international context implies gathering data from participants from different countries with different cultural backgrounds. Therefore, when projects include neuropsychological testing, culture becomes an important factor influencing the performance of participants ${ }^{(5)}$.

According to the United Nations Educational, Scientific and Cultural Organization (UNESCO) ${ }^{(6)}$, culture is defined as 'set of distinctive spiritual, material, intellectual and emotional features of society or a social group, that encompasses, not only art and literature, but lifestyles, ways of living together, value systems,

Disclaimer: This paper was published as part of a supplement to the British Journal of Nutrition, publication of which was supported partially by UNILEVER, NUTRIMENTHE EU Project and an unrestricted educational grant from the University of Granada. The papers included in this supplement were invited by the Guest Editor and have undergone the standard journal formal review process. They may be cited.

Abbreviations: CHOP, Childhood Obesity Project; NGDB, NUTRIMENTHE Global Database; NUHEAL, Nutraceuticals for a Healthy Life. 
traditions and beliefs' (page 1) and cultural neuroscience as 'an emerging research discipline that investigates cultural variation in psychological, neural, and genomic processes as a means of articulating the bidirectional relationship of these processes and their emergent properties, ${ }^{,(7)}$. A growing number of studies showed neuropsychological differences among people from different cultures ${ }^{(5,8)}$. Differences in neuropsychological performance were found among healthy people from different cultures such as Asian $^{(9,10)}$, Hispanic ${ }^{(11,12)}$ or African ${ }^{(13-15)}$. These differences have been shown in verbal and non-verbal tests ${ }^{(16,17)}$, at any age ${ }^{(10,18)}$, and also in brain-damaged patients ${ }^{(19,20)}$. However, some studies failed to detect neuropsychological differences depending on the cultural background ${ }^{(21)}$.

Cultural differences in neuropsychological performance have also been found between persons living in the same country but belonging to different ethnic groups. Manly et al. showed differences between Caucasians and African Americans in cognitive functioning ${ }^{(22-24)}$. Similar differences have been found in Hispanic American samples ${ }^{(22,25,26)}$. Differences have been found even among people from the same ethnicity sharing the same language but living in different countries. For example, Buré-Reyes et al. ${ }^{(27)}$ found differences in memory neuropsychological tests among Hispanics living in different countries (North America, Chile, Santo Domingo and Spain).

The cultural differences in neuropsychological performance have traditionally been mainly explained by variables such as sex, language and ethnicity ${ }^{(19,28-30)}$. When variables such as language, reading ability and analphabetism, bilingualism or socioeconomic status are controlled for, the size of differences in neuropsychological performance decreases ${ }^{(31,32)}$. Therefore, acculturation and quality of education could explain at least part of the cultural differences in neuropsychological performance ${ }^{(23,33)}$.

Nevertheless, recently, new variables related to the modulating effect of culture on the cognitive system have been proposed $^{(34)}$. Agranovich et al. ${ }^{(35)}$ studied the differences between American and Russian adults in time attitudes, and how these can explain the cultural differences in the chronometric neuropsychological tests. Ouellet et al. ${ }^{(36)}$ have shown that time runs from left (past time) to right (future time) for Spaniards but it runs in the opposite direction for Israeli people, reflecting the direction of reading and writing. Fasfous et $a l^{(37)}$ showed that cognitive processes requiring carrying out a culture-free intelligence test may be different, depending on the subject's cultural background.

Despite all evidence about the influence of culture in neuropsychological performance of adults, little is known about the influence of culture in neuropsychological testing in children. In a review, Byrd et al. ${ }^{(38)}$ found that only ten studies about cross-cultural neuropsychology in children were conducted, and, half of them conducted in North America. Most of the studies found differences among children with different cultures. Recently, studies focused on specific cognitive processes. Sobeh \& Spijkers ${ }^{(39)}$ found differences between German and Syrian children in an attention battery, with the latter scoring less than the former, and Kail et $a l^{(40)}$ found differences in speed processing between US and East Asian children. Similar results have been found when children from Sweden, Spain, Iran and China were compared in an executive function inventory ${ }^{(41)}$ or using the Children Colour Trail Test ${ }^{(42)}$ in Moroccan children ${ }^{(43)}$.

However, most of the studies conducted in children have focused on a specific cognitive function but have not used a comprehensive neuropsychological battery. Also, we have not found studies comparing different European countries. The objectives of this study were to compare the neuropsychological performance of children from six European countries (Belgium, Germany, Hungary, Italy, The Netherlands, Poland and Spain) using a comprehensive neuropsychological battery, and applying a statistical procedure to reduce the influence of country/cultural differences in neuropsychological performance.

In the present study, country and culture will be used in an equivalent way. According to the previous definition of culture, several cultures may exist inside one country or several countries could share the same culture. However, in our study, each country is considered to have its own culture. The main objective of this study was to analyse the influence of culture on neuropsychological performance of healthy European children, who were participating in nutrition studies. Also, we were interested in studying the utility of standardisation by country and sex as a way to reduce or eliminate the influence of culture on neuropsychological scores.

\section{Methods}

\section{Study design and participants}

The data obtained to develop this study comes from the NUTRIMENTHE EU Project, which has been previously described in Anjos $e$ $a l{ }^{(1)}$. Within NUTRIMENTHE EU Project (grant agreement no. 212652) framework, the NUTRIMENTHE Global Database (NGDB) has been developed joining the data sets from three different follow-up European cohorts, the Childhood Obesity Project (CHOP) study (Belgium, Germany, Italy, Poland and Spain), the Generation $\mathrm{R}$ study (The Netherlands) and the Nutraceuticals for a Healthy Life (NUHEAL) study (Germany, Hungary and Spain). Description of participants of these cohorts has been already published elsewhere ${ }^{(44-46)}$. After combining the common variables from the three studies, a new cohort was formed with a total of 1050 children who were assessed using a common neuropsychological procedure; all data sets from these children were included in the NGDB.

A common set of questions, anthropometrical examinations, blood parameters, nutritional and baseline characteristic data were collected besides the common neuropsychological test battery with similar methodologies to be able to combine the data sets for analysis. The merging was performed using the statistical programming language R (The R Foundation for Statistical Computing). To embed, recode and standardise all variables in a good, documented and structured way into one common global database has been essential to ensure proper data analysis later on.

In the present analysis, 880 healthy children from six European countries (Belgium, $n$ 63; Germany, $n$ 117; Italy, $n$ 100; The Netherlands, $n$ 199; Poland, $n 102$; and Spain, $n$ 299) were included. All participants from Hungary were excluded because of a significant drop-out rate. In addition, participants without all neuropsychological scores were excluded because 
of technical problems on the computerised tests. Exclusively for the Stroop Test and Hungry Donkey Test (HDT), 681 children were analysed because of the fact that the Dutch children had not finished learning to read at the time of test administration. All participants started primary school at 6 years old. CHOP children were evaluated at 8 years, those from Generation R at 7 years and those participating in the NUHEAL study at 7.5 and 9 years. This study was conducted according to the guidelines laid down in the Declaration of Helsinki, and all procedures involving human subjects were approved by the Ethical Committees of all centres involved in the study. Written informed consent was obtained from all subjects before their inclusion in the study. Characteristics of the study population are listed in Table 1.

\section{Description of the NUTRIMENTHE Neuropsychological Battery}

No neuropsychological tests were available with versions for all participating countries, (Belgium, Germany, Italy, The Netherlands, Poland and Spain). Thus, to assess the child's neurocognitive development, a comprehensive neuropsychological battery was specifically developed for the NUTRIMENTHE project: the NUTRIMENTHE Neuropsychological Battery (NNB). Criteria used to choose the neuropsychological tests were as follows: (1) tests to assess the main neuropsychological domains; (2) tests appropriate for 7-9 year-old children; (3) tests with low verbal stimulus in order to reduce the influence of language; (4) when possible, tests with reduced cultural influence. Most of the tests identified were not available in many of the countries participating in the NUTRIMENTHE project. When a chosen test was not available in one country, procedures were put in place to translate and culturally adapt the test. Finally, the NNB was culturally adapted and translated into six languages and implemented in six European countries. A back-translation procedure was followed to translate the tests. First, instructions were translated from Spanish to each language by a translator, and another expert translated the tests from each language to Spanish. Both versions were compared and discrepancies were sorted out.

The cognitive tests' battery consists of fifteen tests to assess seven domains ${ }^{(47-59)}$ : processing speed, perception, motor, memory, attention, language and executive functions, which are described in Table 2 (full description in the online Supplementary Material). For each subtest, we calculated sex and country internal $z$-scores. Finally, neuropsychological data from 880 European children were used in the statistical analyses included in the present study.

Selected tests were culturally adapted and translated into six languages (Spanish, German, Italian, French, Dutch and Polish) by experts in the field, after obtaining approval from the owners of the copyrights; in addition, licences were obtained for all children to be assessed in the six countries. Tests were administered by health professionals (most of them psychologists) in each country who had been previously trained centrally by the University of Granada, and provided with Standard Operating Procedure (SOP) Manuals, which also included the assessment conditions (i.e. a quiet room without interruptions, without any other person in the room, and conducting the assessment during the afternoon); The training sessions were carried

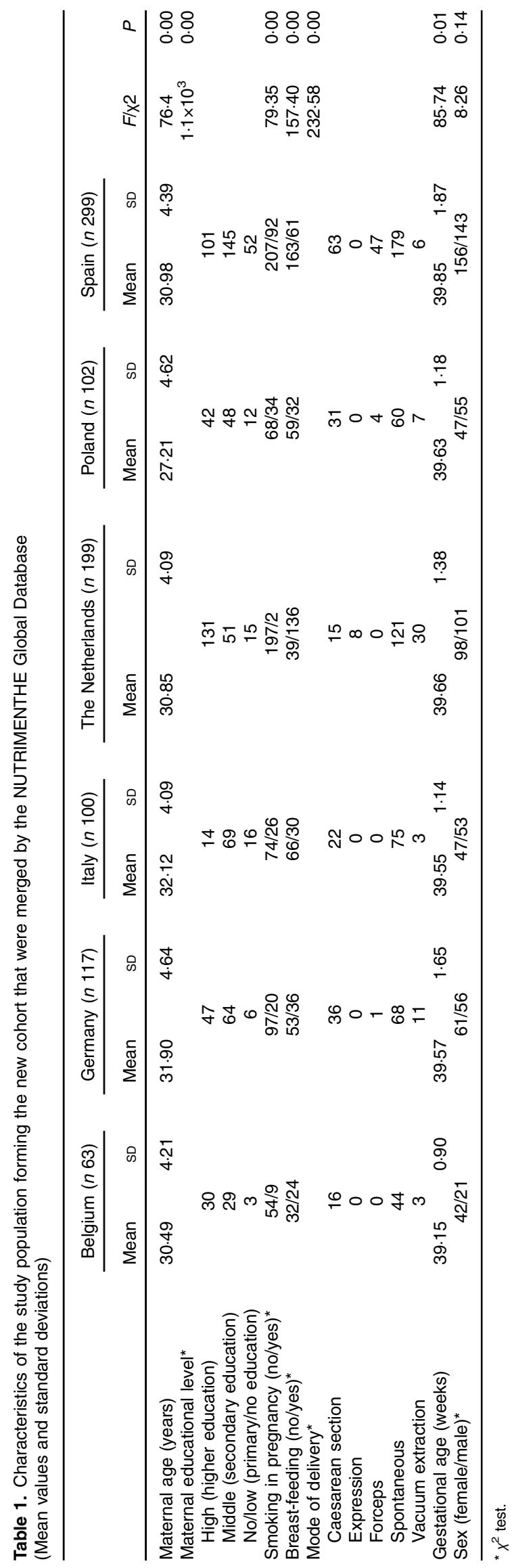


Table 2. NUTRIMENTHE Neuropsychological Battery: test description

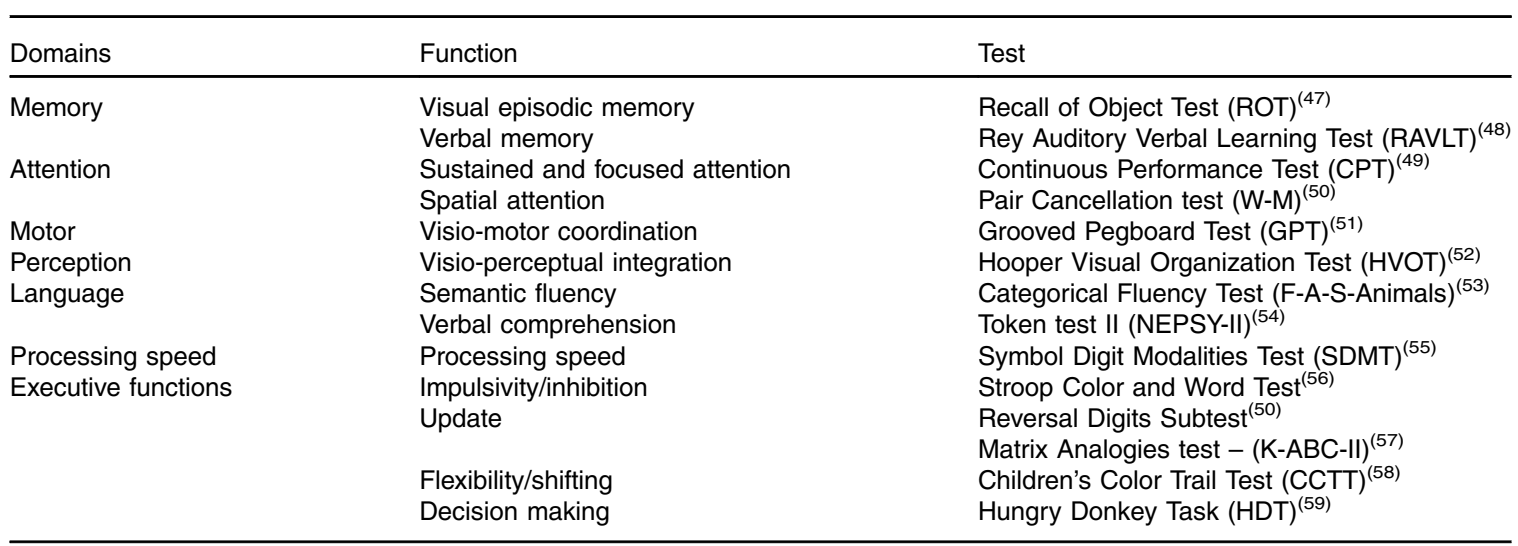

out through several face-to-face meetings organised within the NUTRIMENTHE framework, where different quality control skills were demonstrated under the supervision of an expert from the University of Granada, until all professionals involved revealed very good inter-subject reliability (intraclass correlation coefficient $>0.70$ in all cases). All queries were answered and agreements reached regarding the characteristics of each country for the interpretation of the tests. A NUTRIMENTHE blog containing all SOP was placed in the intranet of the NUTRIMENTHE website, where all researchers could ask questions and provide solutions to problems easily as required.

\section{Statistical analysis}

All scores were entered in the NGDB, consisting of the raw scores of the dependent variables obtained from the neuropsychological tests. Each individual score was subtracted from the corresponding country and sex mean and divided by the corresponding country and sex standard deviation in order to obtain the standardisation by country and sex. This standardisation was performed to reduce effect and noise due to countries. 'Leaving-one-out' standardisation (each observation was standardised using the mean and standard deviation of the particular sample excluding the individual score) was performed in order to eliminate probable effects of extreme values. As there were no differences between the results obtained after applying 'leaving-one-out' and normal standardisation, 'leaving-one-out' standardised scores were not considered in the present analysis. To reduce the number of statistical analyses, a reduced set of dependent variables was selected.

In order to study differences in neuropsychological performance among countries, a one-way ANOVA and KruskalWallis (skewed variables) analysis were conducted using country as a factor and the raw neuropsychological variables as dependent variables. To study the importance of the country variable as a confounder, ordinal logistic regressions were conducted over the outcomes (neurodevelopmental tests), using the available common variables related with neuropsychological performance in children such as sex, type of delivery, maternal age, gestational age and maternal educational level as predictors; raw neuropsychological scores were considered as dependent variables.

The database was imputed using machine-learning techniques; the best suitable one found was missforest (package 'missforest' in R; from Daniel J. Stekhoven, 2013), obtaining an acceptable out-of-bag error of $0 \cdot 07$. The percentage of imputations range from $28 \%$ for HDT to $<1 \%$ for Reversal Digits.

Database set up, including file merging, mistake detection and metadata, and the whole statistical analysis were conducted using STATA 12.1 .

\section{Results}

General characteristics of the children by country included in the present analysis (Table 1).

Maternal age was the youngest in Poland, and the oldest in Italy. The percentage of mothers with a high level of education was higher than $50 \%$ in the Netherlands and Germany; Germany and Italy were the countries with the highest percentage of mothers with a medium level of education $(>70 \%)$. The major percentage of mothers with a low level of education came from Spain and Italy ( $>17 \%$ ). In all, $22 \%$ of the mothers were smokers during pregnancy and $44 \%$ of women breast-fed their babies (with a higher percentage in the Netherlands (78\%) and the lowest in Spain (27\%)). A total of $64 \%$ of the babies had a spontaneous mode of delivery. The instrumental deliveries represented the other 36\%; from these, $21 \%$ of the mothers delivered by caesarean section, with this type of delivery being more frequent in Germany (31\%), Poland (30\%), Italy (22\%) and Spain (21\%) compared with the other countries. Delivery by forceps represented $16 \%$ of all deliveries registered in the Spanish sample, compared with a minimal incidence in Poland (4\%) and Germany (0.85\%), and none reported in the rest of the countries. Vacuum extraction was the mode of delivery in $26 \%$ of the Dutch mothers and $6 \%$ of the German ones; in the rest of the countries, vacuum extraction had a very low incidence.

All children were born at term, although there were differences between countries, with the Belgian children being the youngest ones. In all, 451 children were girls and 429 were 


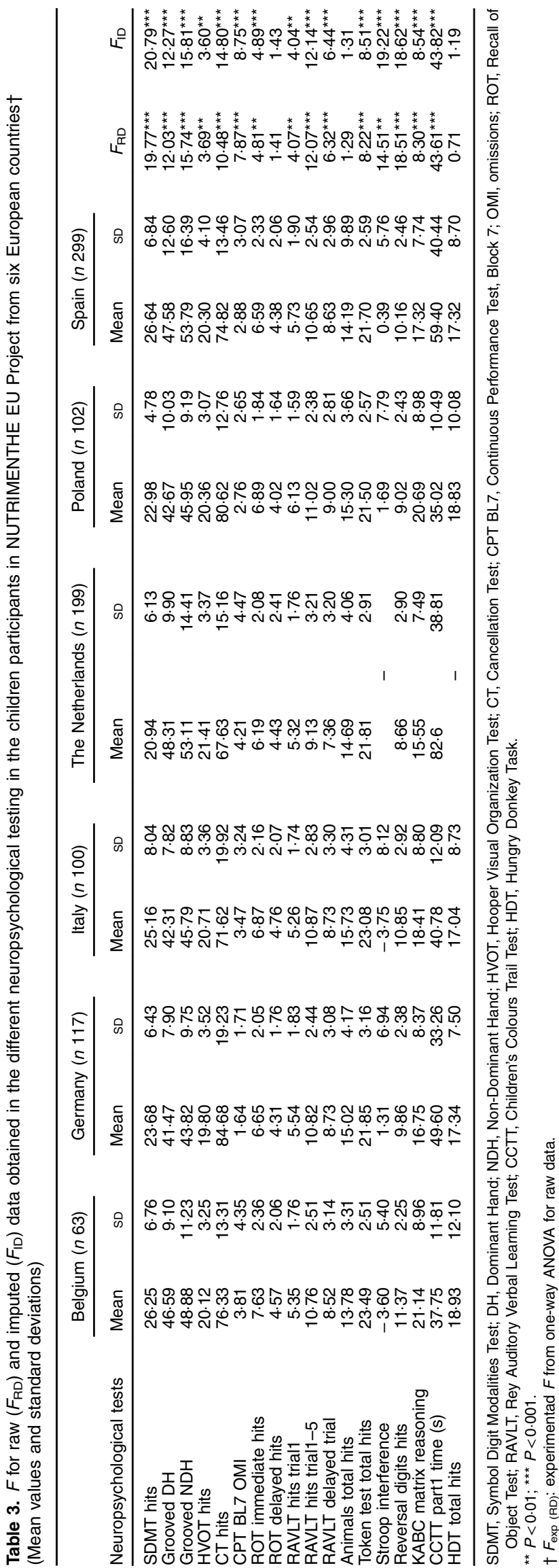

boys. No statistical differences in the females-males distribution between countries were found.

\section{Neuropsychological differences among countries}

As shown in Table 3, significant statistical differences were found among the six countries in all the neuropsychological variables, except for Recall of Object Test delayed hits, Animal total hits and HDT total hits. In general, the Netherlands' children scored higher than those from the other countries. Same results were obtained with the multiple imputed scores (Table 3).

As described in the statistical methods section, scores were standardised by country and sex. After that, the previous statistical differences among countries disappeared $(P>0 \cdot 8)$, which is congruent with the standardisation procedure.

\section{Predictors of the neuropsychological differences between countries}

In order to study the importance of the country variable as a confounder, raw neuropsychological scores were adjusted using the available confounder variables related to neuropsychological performance in children, such as sex, breast-feeding, type of delivery, maternal age, gestational age, maternal educational level and smoking. After adjusting for these confounders, the country became the main predictor in most of the neuropsychological variables for raw data (Table 4).

\section{Discussion}

The main objective of this study was to analyse the influence of culture (comparing different countries) on the neuropsychological performance of healthy European children. Also, we were interested in studying the utility of standardisation by country and sex as a way to reduce the culture influence. As expected, results showed differences in the neuropsychological performance among children of the six countries involved. Cultural differences related to neuropsychological performance remained after adjusting for other confounders such as sex, maternal age, maternal educational level, smoking during pregnancy, type of delivery and gestational age. Finally, differences among countries disappeared and influence of the country was considerably reduced when standardised scores by country and sex were used.

As previously reported ${ }^{(5,38)}$, culture influenced neuropsychological performance of children. However, there were no previous studies comparing neuropsychological performance of European children, nor on the variables that could explain differences among cultures. Besides traditional variables such as language, educational system or socioeconomic status, which could account for a part of the variability ${ }^{(23,33)}$, country should be considered an important confounder in multinational studies or in intra-national studies comparing different ethnicities, when neuropsychological performance is measured. This may be due to the fact that the 'culture/country' variable independently influences the above-mentioned variables in neurodevelopment.

It is worth noting that differences among countries in neuropsychological performance are not homogeneous. The 
Table 4. Raw and imputed data from ordered logistic regression of the different tests performed in the NUTRIMENTHE children with the available confounders from six European countries $\dagger$ (Coefficients of the ordered logistic regression model for imputed data and $95 \%$ confidence intervals)

\begin{tabular}{|c|c|c|c|c|c|c|c|c|}
\hline \multirow[b]{2}{*}{ Neuropsychological tests } & \multicolumn{2}{|c|}{ Study country } & \multicolumn{2}{|c|}{ Sex } & \multicolumn{2}{|c|}{ Maternal age } & \multicolumn{2}{|c|}{ Maternal educational level } \\
\hline & Coefficient & $95 \% \mathrm{Cl}$ & Coefficient & $95 \% \mathrm{Cl}$ & Coefficient & $95 \% \mathrm{Cl}$ & Coefficient & $95 \% \mathrm{Cl}$ \\
\hline SDMT hits & $0.09^{\star \star}$ & $0.02,0.17$ & $0.61^{\star \star \star}$ & $0.37,0.84$ & $0.03^{\star}$ & $0.004,0.05$ & -0.03 & $-0.08,0.01$ \\
\hline Grooved DH & $0 \cdot 14^{\star \star \star}$ & $0.06,0.21$ & $-0.33^{\star *}$ & $-0.56,-0.10$ & -0.01 & $-0.03,0.01$ & -0.03 & $-0.08,0.015$ \\
\hline Grooved NDH & $0.21^{\star * *}$ & $0.14,0.28$ & -0.04 & $-0.27,0.19$ & -0.17 & $-0.04,-0.009$ & -0.01 & $-0.06,0.03$ \\
\hline HVOT hits & $0.09^{\star \star}$ & $0.01,0.16$ & $0.45^{\star \star \star}$ & $0.21,0.68$ & $0.04^{\star \star}$ & $0.01,0.06$ & $0.22^{\star \star \star}$ & $0.16,0.27$ \\
\hline CT hits & $-0.08^{*}$ & $-0.15,-0.008$ & $0.85^{\star \star *}$ & $0.61,1.09$ & $-0.001^{* *}$ & $-0.02,0.02$ & $-0.05^{\star \star}$ & $-0.10,-0.001$ \\
\hline CPT BL7 OMI & -0.02 & $-0.09,0.05$ & -0.07 & $-0.31,0.15$ & -0.002 & $-0.02,0.02$ & $0.10^{\star \star \star}$ & $0.05,0.15$ \\
\hline ROT immediate hits & -0.05 & $-0.13,0.01$ & $0.29^{\star}$ & $0.05,0.52$ & -0.002 & $-0.02,0.02$ & $0.07^{\star \star \star}$ & $0.02,0.12$ \\
\hline ROT delayed hits & -0.16 & $-0.08,0.05$ & $0.32^{\star *}$ & $0.07,0.54$ & 0.01 & $-0.01,0.04$ & 0.01 & $-0.03,0.07$ \\
\hline RAVLT hits trial1 & $0.08^{*}$ & $0.008,0.15$ & 0.20 & $-0.02,0.44$ & $-0.02^{*}$ & $-0.05,0.007$ & $0.05^{*}$ & $-0.001,0.10$ \\
\hline RAVLT hits trial1-5 & -0.049 & $-0.12,0.02$ & $0.46^{* * *}$ & $0.23,0.70$ & $-0.03^{*}$ & $-0.05,-0.005$ & 0.002 & $-0.04,0.05$ \\
\hline RAVLT delayed trial & -0.02 & $-0.90,0.04$ & $0.43^{\star \star *}$ & $0.19,0.66$ & -0.02 & $-0.05,0.001$ & 0.004 & $-0.04,0.05$ \\
\hline Animals total hits & $-0.08^{\star \star}$ & $-0.15,-0.01$ & $0 \cdot 10$ & $-0.12,0.34$ & 0.01 & $-0.007,0.04$ & 0.02 & $-0.25,0.07$ \\
\hline Token test total hits & $-0 \cdot 15^{\star \star \star}$ & $-0.22,-0.07$ & $0.32^{\star \star}$ & $0.09,0.55$ & $0.06^{\star * \star}$ & $0.03,0.09$ & 0.02 & $-0.02,0.07$ \\
\hline Stroop interference & $0.19^{\star \star \star}$ & $0.12,0.27$ & 0.11 & $-0.11,0.34$ & 0.002 & $-0.02,0.02$ & 0.04 & $-0.002,0.09$ \\
\hline Reversal digits hits & $-0 \cdot 10^{\star \star \star}$ & $-0.17,0.02$ & 0.11 & $0.12,0.34$ & $0.04^{* \star *}$ & $0.01,0.06$ & -0.04 & $-0.09,0.004$ \\
\hline K-ABC-II matrix reasoning & -0.19 & $-0.09,0.05$ & $0.23^{*}$ & $0.005,0.49$ & 0.01 & $-0.007,0.04$ & 0.04 & $-0.009,0.09$ \\
\hline CCTT part1 time (s) & $0.15^{\star \star \star}$ & $0.08,0.23$ & -0.11 & $-0.35,0.11$ & 0.008 & $-0.01,0.03$ & $-0 \cdot 10^{\star \star \star}$ & $-0.16,-0.05$ \\
\hline \multirow[t]{3}{*}{ HDT total hits } & -0.06 & $-0.13,0.01$ & $-0 \cdot 10$ & $-0.33,0.12$ & 0.02 & $-0.004,0.04$ & $0.06^{\star \star}$ & $0.01,0.11$ \\
\hline & \multicolumn{2}{|c|}{ Smoking in pregnancy } & \multicolumn{2}{|c|}{ Mode of delivery } & \multicolumn{2}{|c|}{ Gestational age } & \multicolumn{2}{|c|}{ Breast-feeding } \\
\hline & Coefficient & $95 \% \mathrm{Cl}$ & Coefficient & $95 \% \mathrm{Cl}$ & Coefficient & $95 \% \mathrm{Cl}$ & Coefficient & $95 \% \mathrm{Cl}$ \\
\hline SDMT hits & 0.22 & $-0.07,0.53$ & -0.04 & $-0.13,0.04$ & 0.03 & $-0.04,0.10$ & $-0.31^{\star *}$ & $-0.56,-0.05$ \\
\hline Grooved DH & -0.07 & $-0.38,0.22$ & -0.04 & $-0.56,-0.10$ & $-0.14^{\star}$ & $-0.21,-0.06$ & 0.15 & $-0.09,0.41$ \\
\hline Grooved NDH & -0.25 & $-0.56,0.05$ & -0.08 & $-0.15,0.03$ & -0.05 & $-0.13,0.03$ & 0.06 & $-0.18,0.32$ \\
\hline HVOT hits & 0.05 & $-0.25,0.35$ & 0.04 & $-0.04,0.13$ & 0.02 & $-0.05,0.10$ & 0.21 & $-0.03,0.46$ \\
\hline CT hits & $0.35^{\star *}$ & $0.04,0.66$ & -0.08 & $-0.17,0.01$ & 0.04 & $-0.02,0.11$ & -0.0002 & $-0.25,0.25$ \\
\hline CPT BL7 OMI & $-0 \cdot 12$ & $-0.42,0.18$ & -0.02 & $-0.12,0.06$ & 0.02 & $-0.04,0.09$ & 0.05 & $-0.19,0.30$ \\
\hline ROT immediate hits & -0.07 & $-0.37,0.21$ & 0.05 & $-0.03,0.14$ & 0.03 & $-0.03,0.11$ & $-0.39^{\star \star}$ & $-0.65,-0.14$ \\
\hline ROT delayed hits & -0.18 & $0.48,0.12$ & 0.04 & $-0.05,0.13$ & -0.02 & $-0.10,0.04$ & 0.09 & $-0.15,0.39$ \\
\hline RAVLT hits trial1 & 0.01 & $-0.29,0.32$ & 0.008 & $-0.08,0.10$ & 0.07 & $-0.013,0.15$ & -0.23 & $-0.48,0.02$ \\
\hline RAVLT hits trial1-5 & 0.05 & $-0.24,0.35$ & -0.06 & $-0.16,0.02$ & 0.07 & $-0.001,0.14$ & $-0.34^{\star \star \star}$ & $-0.60,-0.09$ \\
\hline RAVLT delayed trial & -0.18 & $-0.48,0.10$ & -0.02 & $-0.11,0.07$ & 0.06 & $-0.003,0.13$ & $-0.29^{\star}$ & $-0.54,-0.03$ \\
\hline Animals total hits & -0.004 & $-0.30,0.29$ & -0.04 & $-0.13,0.04$ & 0.01 & $-0.05,0.09$ & 0.17 & $-0.08,0.42$ \\
\hline Token test total hits & -0.08 & $-0.38,0.21$ & -0.001 & $-0.09,0.09$ & 0.03 & $-0.04,0.10$ & -0.003 & $-0.25,0.25$ \\
\hline Stroop interference & -0.01 & $-0.32,0.30$ & 0.01 & $-0.07,0.11$ & 0.007 & $-0.06,0.07$ & $0.51^{\star \star \star}$ & $0.25,0.76$ \\
\hline Reversal digits hits & 0.11 & $-0.18,0.42$ & -0.02 & $-0.11,0.06$ & $0.07^{\star}$ & $0.002,0.15$ & -0.23 & $-0.48,0.02$ \\
\hline K-ABC-II matrix reasoning & 0.009 & $-0.30,0.30$ & -0.03 & $-0.13,0.05$ & 0.07 & $-0.006,0.15$ & -0.04 & $-0.29,0.21$ \\
\hline CCTT part1 time (s) & $-0.45^{\star \star \star}$ & $-0.75,-0.15$ & 0.03 & $-0.05,0.13$ & $-0.15^{\star \star \star}$ & $-0.24,-0.07$ & $0.75^{\star \star \star}$ & $0.49,1.01$ \\
\hline HDT total hits & -0.22 & $-0.53,0.07$ & 0.01 & $-0.07,0.10$ & 0.02 & $-0.06,0.07$ & $0.39^{\star *}$ & $0.14,0.65$ \\
\hline
\end{tabular}

SDMT, Symbol Digit Modalities Test (total hits); DH, Dominant Hand; NDH, Non-Dominant Hand; HVOT, Hooper Visual Organization Test (total hits); CT, Cancellation Test (total hits); CPT BL7, Continuous Performance Test (total hits), Block 7; OMI, omissions; ROT, Recall of Object Test (immediate and delayed recalled pictures); RAVLT, Rey Auditory Verbal Learning Test (recalled words in trial 1, trial 1-5 and delayed (hits)); K-ABC-II, Matrix Analogies Test (total hits); CCTT, Children's Colors Trail Test (time part 1 (s)); HDT, Hungry Donkey Task (total score).

$P<0.01 ; * * * \quad P<0.001$

† Children's neuropsychological scores adjusted by study country, sex, maternal age , maternal educational level, smoke in pregnancy, mode of delivery, gestational age and breast-feeding. 
largest differences have been found in processing speed and executive functions and the smallest in the delayed recall of visual memory, verbal fluency and decision making. Specific neuropsychological patterns for specific cultures have not been demonstrated, but differences have been reported in both verbal $^{(60)}$ and non-verbal ${ }^{(29,37)}$ tests. Our results are congruent with previous studies showing cultural differences in several cognitive functions ${ }^{(38)}$. Also, it should be mentioned that most of the studies compared neuropsychological execution only between $2-3$ cultures $^{(41)}$; studies with six cultures/countries are inexistent. In that sense, NNB is the first European neuropsychological battery for children designed in such way as to be used in six different countries. The development of this tool permitted us to build up the NGDB, giving us the opportunity to evaluate cultural effects in a population of European children.

Standardisation by country and sex has been useful to eliminate differences among countries. This is due to the fact that standardisation sorts neuropsychological performance inside each country according to the country means. This can be done under the assumption that groups of children in each country are normal children, and thus, they represent the normal variability. Under this assumption, a higher score in the same neuropsychological test of one child from one country when compared with that of another child from another country does not indicate a better execution.

Those results may have important implications for future research. First, they highlight the need for developing procedures to compare neuropsychological performance among children from different cultures. This is important even when comparing children inside the same country, with the same education system but different culture. In the case of Europe, this is almost mandatory because of the number of multinational studies promoted by the European Commission in which neuropsychological assessment is involved. Also, it enhances the importance of obtaining 'normal' reference values for neuropsychological performance in each European country considering minorities living inside the country.

Our results have some limitations. First, the unbalanced sample sizes in the three studies included in the database prevented this study from using data as a reference for each test in each country. Second, the number of children from each country was different and, in some countries, was relatively small. The objective of this study was not to obtain representative normal reference values for each country, but our conclusions for countries should be considered with caution. Despite the unbalanced situation, comparison between countries could be made, and in many cases the results were clearly significant; hence, we believe that this unbalance does not have a strong influence on the main results of this study. Furthermore, the potential approaches to differentiate useful variables from noisy ones, as well as to detect patterns of association between selected variables, demonstrates the need for further studies to determine the usefulness of the shorter and more targeted assessments. Our study has been conducted only in a selected age range (7-9 years); thus, future studies should be conducted to also explore whether our results can be extended to other ages. Finally, it should be considered that neuropsychological tests were administrated by different technicians with different backgrounds. This variable could increase differences among countries. However, common training carried $\backslash$ out by the same person was provided to all technicians in order to decrease the influence of this effect. Also, our study cannot verify which country/cultural variables such as language, educational system, etc., are involved in the neuropsychological differences. A crucial aspect of explanatory statistical inference in this context is that we need methods that allow us to deal with categorical outcomes and to include a large number of potentially correlated predictors while avoiding over fitting.

The strengths of the present study have been the development and application of a common Neuropsychological Battery, translated into eight languages, and assessed in 880 European children, which can serve as a reference for future studies. The NGDB allowed us to pool results of three cohort studies that use different tests in assessing the same phenotypes. The majority of functional domains have been divided into a set of specific subdomains. We emphasise that a cautious and robust approach was needed in order to combine the data in a meaningful way, particularly in pooled analyses, where a priori theoretical background and statistical modelling has been employed. Sensible combinations of data, originated from the different neuropsychological tests, have been driven by theoretical considerations of the likely specificity of the effects of particular nutritional and environmental agents on neuropsychological development. This will allow practitioners to gain a clear understanding about the better assessment methods to be used when limited resources and time are available in applied clinical settings.

It is notable that very few neuropsychologists work in the field of nutrition. The presence of these professionals with a background in both neurodevelopment and neuropsychological development is critical to the elaboration and application of assessment protocols (on the basis of their knowledge of brain development and neuropsychological testing), as well as quality control on the data collection and analysis, and for the interpretation of the study findings. Their inclusion in multidisciplinary research teams will improve the quality of research in this important field.

In summary, it is well known that culture is an important confounding factor in neuropsychological testing. In the present study, statistical differences in neuropsychological performance among children of six European countries were demonstrated; those differences remained even after standardisation of the test scoring and adjusting for other confounders related to neuropsychological execution, such as maternal education or mode of delivery. Statistical differences among countries disappeared when standardised scores by country and sex were used. We believe that these findings are of major importance for further studies and can be considered beyond its limitations. Future research should determine what variables can justify those differences and which ones should be tested in future projects, when the use or development of new neuropsychological batteries for multicountry assessment is planned.

\section{Acknowledgements}

The authors thank all participating children for their collaboration and all colleagues in the study centres for their support. 
The studies reported herein have been carried out with financial support from the Commission of the European Communities, specific Research and Technological Development Programme 'Quality of Life and Management of Living Resources', within the 7th Framework Programme (FP7/2008-2013) under grant agreement no. 212652 (NUTRIMENTHE Project: 'The Effect of Diet on the Mental Performance of Children'). The data included in this study come from the neuropsychological development performed in the 1050 European children participants in the CHOP EU project (FOOD-CT-2005-007036), the Generation R study (www.generationr.nl/researchers.html) and the NUHEAL Project: 'Nutraceuticals for a healthy life' (BIOMED QRLT-199900888). This publication is the work of the authors and does not reflect the views of the EU Commission.

All authors helped in the interpretation of results and contributed to manuscript preparation. M. P.-G. was responsible for the neuropsychological battery development; M. P.-G. and C. C. wrote the manuscript; J. d. D. L. performed the statistical analysis; F. J. T.-E., C. M.-Z. and J. S.-d. G. were responsible of neuropsychological evaluation of NUHEAL children at 8.5 years and Generation R children at 7 years, supported all other teams and helped to complete the databases; T. A. worked in the NUTRIMENTHE management team, collaborated in the organisation of the study and the completion of the NUTRIMENTHE Global Database; M. W. and V. G. helped in the development of the NUTRIMENTHE Global Database; F. J. T.-E., C. M.-Z., J. S.-d. G., T. A., M. W., V. G., D. G., E. V., P. P., B. K., J. E., H. T. and T. A. supervised the manuscript. D. G., E. V., P. P., B. K. and J. E. were responsible for the CHOP study in Poland, Italy, Belgium, Germany and Spain, respectively; H. T. was responsible for the Generation $\mathrm{R}$ study in Rotterdam (The Netherlands); T. A. and B. K. were responsible for the NUHEAL study in Hungary and Germany, respectively; C. C. was the coordinator of NUTRIMENTHE EU Project and supervised the NUHEAL study in Granada.

None of the authors has any conflicts of interest to declare.

\section{Supplementary material}

For supplementary material/s referred to in this article, please visit https://doi.org/10.1017/S0007114517000824

\section{References}

1. Anjos T, Altmäe S, Emmett P, et al. (2013) Nutrition and neurodevelopment in children: focus on NUTRIMENTHE project. Eur J Nutr 52, 1825-1842.

2. Smith MA \& Scholey AB (2014) Nutritional influences on human neurocognitive functioning. Front Hum Neurosci 8 , 358.

3. Fretham SJ, Carlson ES \& Georgieff MK (2011) The role of iron in learning and memory. Adv Nutr 2, 112-121.

4. Portillo-Reyes V, Pérez-García M, Loya-Méndez Y, et al. (2014) Clinical significance of neuropsychological improvement after supplementation with omega-3 in 8-12 years old malnourished Mexican children: a randomized, double-blind, placebo and treatment clinical trial. Res Dev Disabil 35, 861-870.
5. Uzzell BP, Ponton M \& Ardila A (editors) (2013) International Handbook of Cross-Cultural Neuropsychology. New York: Psychology Press.

6. United Nations Educational, Scientific and Cultural Organization (UNESCO) (2001) UNESCO Universal Declaration on Cultural Diversity. Paris: UNESCO. http://www.unesco.org/ confgen/press_rel/021101_clt_diversity.shtml

7. Chiao JY (2009) Cultural neuroscience: a once and future discipline. Prog Brain Res 178, 287-304.

8. Ardila A (2007) The impact of culture on neuropsychological test performance. In International Handbook of CrossCultural Neuropsychology, pp. 23-44 [BP Uzzell, M Pontón and A Ardila, editors]. Mahwah, NJ: Lawrence Erlbaum.

9. Hsieh SLJ \& Tori CD (2007) Normative data on cross-cultural neuropsychological tests obtained from Mandarin-speaking adults across the life span. Arch Clin Neuropsychol 22, 283-296.

10. Lan X, Legare CH, Ponitz CC, et al. (2011) Investigating the links between the subcomponents of executive function and academic achievement: a cross-cultural analysis of Chinese and American pre-schoolers. J Exp Child Psychol 108, 677-692.

11. Judd T, Capetillo D, Carrión-Baralt J, et al. (2009) Professional considerations for improving the neuropsychological evaluation of Hispanics: a National Academy of Neuropsychology education paper. Arch Clin Neuropsychol 24, 127-135.

12. Llorente AM (2008) Principles of Neuropsychological Assessment with Hispanics. New York, NY: Springer.

13. Khalil MS (2010) Preliminary Arabic normative data of neuropsychological tests: the verbal and design fluency. J Clin Exp Neuropsychol 32, 1028-1035.

14. Ruffieux N, Njamnshi AK, Mayer E, et al. (2009) Neuropsychology in Cameroon: first normative data for cognitive tests among school-aged children. Child Neuropsychol 16, 1-19.

15. Wicherts JM, Dolan CV \& van der Maas HL (2010) A systematic literature review of the average IQ of sub-Saharan Africans. Intelligence 38, 1-20.

16. Cherner M, Suarez P, Lazzaretto D, et al. (2007) Demographically corrected norms for the Brief Visuospatial Memory Test-revised and Hopkins Verbal Learning Test-revised in monolingual Spanish speakers from the US-Mexico border region. Arch Clin Neuropsychol 22, 343-353.

17. Matute E, Rosselli M, Ardila A, et al. (2004) Verbal and nonverbal fluency in Spanish-speaking children. Dev Neuropsychol 26, 647-660.

18. Park D \& Gutchess A (2006) The cognitive neuroscience of aging and culture. Curr Dir Psychol Sci 15, 105-108.

19. Boone KB, Victor TL, Wen J, et al. (2007) The association between neuropsychological scores and ethnicity, language, and acculturation variables in a large patient population. Arch Clin Neuropsychol 22, 355-365.

20. Ferraro FR \& McDonald LR (2005) More culturally sensitive neuropsychological tests (and normative data) needed. Alzheimer Dis Assoc Disord 19, 53.

21. Ostrosky-Solis F, Gutierrez AL, Flores MR, et al. (2007) Same or different? Semantic verbal fluency across Spanish-speakers from different countries. Arch Clin Neuropsychol 22, 367-377.

22. Byrd DA, Touradji P, Tang MX, et al. (2004) Cancellation test performance in African American, Hispanic, and White elderly. J Int Neuropsychol Soc 10, 401-411.

23. Byrd DA, Sanchez D \& Manly JJ (2005) Neuropsychological test performance among Caribbean-born and US-born African American elderly: the role of age, education and reading level. J Clin Exp Neuropsychol 27, 1056-1069. 
24. Manly JJ, Byrd DA, Touradji P, et al. (2004) Acculturation, reading level, and neuropsychological test performance among African American elders. Appl Neuropsychol 11, 37-46.

25. Puente AE, Perez-García M, Vilar-Lopez R, et al. (2013) Neuropsychological assessment of culturally and educationally dissimilar individuals. In Handbook of Multicultural Mental Health: Assessment and Treatment of Diverse Population, pp. 225-242 [FA Paniagua and A-M Yamada, editors]. San Diego, CA: Academic Press.

26. Salazar GD, Garcia MP \& Puente AE (2007) Clinical neuropsychology of Spanish speakers: the challenge and pitfalls of a neuropsychology of a heterogeneous population. In International Handbook of Cross-Cultural Neuropsychology, pp. 283-302 [BP Uzzell, MO Pontón and A Ardila, editors]. Mahwah, NJ: Lawrence Erlbaum Associates.

27. Buré-Reyes A, Hidalgo-Ruzzante N, Vilar-López R, et al. (2013) Neuropsychological test performance of Spanish speakers: is performance different across different Spanishspeaking subgroups? J Clin Exp Neuropsychol 35, 404-412.

28. Ardila A (2005) Cultural values underlying psychometric cognitive testing. Neuropsychol Rev 15, 185-195.

29. Rosselli M \& Ardila A (2003) The impact of culture and education on non-verbal neuropsychological measurements: a critical review. Brain $\operatorname{Cogn}$ 52, 326-333.

30. Gasquoine PG (2009) Race-norming of neuropsychological tests. Neuropsychol Rev 19, 250-262.

31. Brickman AM, Cabo R \& Manly JJ (2006) Ethical issues in crosscultural neuropsychology. Appl Neuropsychol 13, 91-100.

32. Schwartz BS, Glass TA, Bolla KI, et al. (2004) Disparities in cognitive functioning by race/ethnicity in the Baltimore Memory Study. Environ Health Perspect 112, 314-320.

33. Manly JJ, Jacobs DM, Touradji P, et al. (2002) Reading level attenuates differences in neuropsychological test performance between African American and White elders. J Int Neuropsychol Soc 8, 341-348.

34. Wilson M (2010) The re-tooled mind: how culture re-engineers cognition. Soc Cogn Affect Neurosci 5, 180-187.

35. Agranovich AV, Panter AT, Puente AE, et al. (2011) The culture of time in neuropsychological assessment: exploring the effects of culture-specific time attitudes on timed test performance in Russian and American samples. I Int Neuropsychol Soc 17, 692-701.

36. Ouellet M, Santiago J, Israeli Z, et al. (2010) Is the future the right time? Exp Psychol 57, 308-314.

37. Fasfous AF, Hidalgo-Ruzzante N, Vilar-López R, et al. (2013) Cultural differences in neuropsychological abilities required to perform intelligence tasks. Arch Clin Neuropsychol 28, 784-790.

38. Byrd D, Arentoft A, Scheiner D, et al. (2008) State of multicultural neuropsychological assessment in children: current research issues. Neuropsychol Rev 18, 214-222.

39. Sobeh J \& Spijkers W (2013) Development of neuropsychological functions of attention in two cultures: a cross-cultural study of attentional performances of Syrian and German children of pre-school and school age. Eur J Dev Psychol 10, 318-336.

40. Kail RV, McBride-Chang C, Ferrer E, et al. (2013) Cultural differences in the development of processing speed. Dev Sci 16, 476-483.
41. Thorell LB, Veleiro A, Siu AF, et al. (2013) Examining the relation between ratings of executive functioning and academic achievement: findings from a cross-cultural study. Child Neuropsychol 19, 630-638.

42. Llorente AM, Williams J, Satz P, et al. (2003) Children's Colour Trails Test-Professional Manual. Lutz, FL: Psychological Assessment Resources.

43. Fasfous AF, Puente AE, Pérez-Marfil MN, et al. (2013) Is the colour trails culture free? Arch Clin Neuropsychol 28, 743-749.

44. Schiess S, Grote V, Scaglioni S, et al. (2010) Introduction of complementary feeding in 5 European countries. J Pediatr Gastroenterol Nutr 50, 92-98.

45. Jaddoe VW, Mackenbach JP, Moll HA, et al. (2006) The Generation R Study: design and cohort profile. Eur $J$ Epidemiol 21, 475-484.

46. Krauss-Etschmann S, Shadid R, Campoy C, et al. (2007) Effects of fish-oil and folate supplementation of pregnant women on maternal and fetal plasma concentrations of docosahexaenoic acid and eicosapentaenoic acid: a European randomized multicenter trial. Am J Clin Nutr 85, 1392-1400.

47. Snodgrass JG \& Vanderwart M (1980) A standardized set of 260 pictures: norms for name agreement, image agreement, familiarity, and visual complexity. J Exp Psychol Hum Learn $\mathbf{6}$, 174-215.

48. Rey A (1941) L'examen psychologique dans les cas d'encephalopathie trau-matique: the Rey Auditory Verbal Learning Test [RVLMT] (The psychological examination in cases of head trauma: the Rey Auditory Verbal Learning Test [RVLMT]). Arch Psychol (Geneve) 28, 215-285.

49. Conners CK, Epstein JN, Angold A, et al. (2003) Continuous performance test performance in a normative epidemiological sample. J Abnorm Child Psychol 31, 555-562.

50. Woodcock R, McGrew K \& Mather N (2001) WoodcockJohnson III. Tests of Cognitive Abilities. Rolling Meadows, IL: Riverside Publishing.

51. Lafayette I (2002) Grooved Pegboard User Instructions. Lafayette, IN: Lafayette Instruments.

52. Hooper H (1983) Hooper Visual Organization Test Manual. Los Angeles, CA: WPS.

53. Strauss E, Sherman EM \& Spreen O (2006) A Compendium of Neuropsychological Tests: Administration, Norms, and Commentary. New York: Oxford University Press.

54. Korkman M, Kirk U \& Kemp S (2007) NEPSY II. Administration Manual. San Antonio, TX: Pearson Assessment.

55. Smith A (2007) Symbol Digit Modalities Test, 10th printing ed. Los Angeles, CA: WPS

56. Golden C (1978) Stroop Color and Word Test. Chicago, IL: Stoelting Company.

57. Kaufman AS \& Kaufman NL (2004) Kaufman Assessment Battery for Children, 2nd ed. San Antonio, TX: Pearson.

58. Llorente A, Williams J, Satz P, et al. (2003) Children's Color Trail Test. Lutz, FL: Psychological Assessment Resources, Inc.

59. Crone EA \& Van der Molen MW (2004) Developmental changes in real life decision making: performance on a gambling task previously shown to depend on the ventromedial prefrontal cortex. Dev Neuropsychol 25, 251-279.

60. Portocarrero JS, Burright RG \& Donovick PJ (2007) Vocabulary and verbal fluency of bilingual and monolingual college students. Arch Clin Neuropsychol 22, 415-422. 